\title{
Materials modeling and development for use in double-exposure lithography applications
}

\author{
Saul Lee \\ Kane Jen \\ C. Grant Willson \\ The University of Texas at Austin \\ Department of Chemical Engineering \\ Austin, Texas 78712
}

\author{
Jeffrey Byers \\ Paul Zimmerman \\ SEMATECH \\ 2706 Montopolis Drive \\ Austin, Texas 78741
}

Nicholas J. Turro

Columbia University

Department of Chemistry

New York, New York 10027

\begin{abstract}
The current optical photolithography technology is approaching the physical barrier to the minimum achievable feature size. To produce smaller devices, new resolution enhancement technologies must be developed. Double-exposure lithography has shown promise as a potential pathway that is attractive because it is much cheaper than double-patterning lithography and can be deployed on existing imaging tools. However, this technology is not possible without the development of new materials with nonlinear response to exposure dose. The performance of existing materials such as reversible contrast enhancement layers (rCELs), and theoretical materials such as intermediate state twophoton (ISTP) and optical threshold layer (OTL) materials in doubleexposure applications have been investigated through computer simulation. All three materials yielded process windows in double-exposure mode. OTL materials showed the largest process window (depth of focus (DOF) $0.14 \mu \mathrm{m}$, exposure latitude (EL) $5.1 \%$ ). ISTP materials had the next-largest process window (DOF $0.12 \mu \mathrm{m}$, EL 3.2\%), followed by the rCEL $(0.11 \mu \mathrm{m}, 0.58 \%)$. This study is an analysis of the feasibility of using the materials in double-exposure mode. () 2009 Society of Photo-Optical Instrumentation Engineers. [DOI: 10.1117/1.3095589]
\end{abstract}

Subject terms: double-exposure lithography; immersion lithography; doublepatterning lithography; resolution enhancement techniques.

Paper 08027SSR received Apr. 30, 2008; revised manuscript received Oct. 15, 2008; accepted for publication Jan. 26, 2009; published online Mar. 6, 2009.

\section{Introduction}

The current technological progression of the photolithography industry has reached a limit in the maximum achievable resolution. Resolution as determined by the half-pitch critical dimension (CD) is limited by the Rayleigh equation

$\mathrm{CD}=\frac{k_{1} \lambda}{\mathrm{NA}}$

where $k_{1}$ is the process aggressiveness factor, $\lambda$ is the wavelength of the imaging tool, and NA is the numerical aperture of the imaging lens. To reduce the half-pitch $\mathrm{CD}$, the industry must reduce $k_{1}$ or $\lambda$, or increase NA. The theoretical minimum value for $k_{1}$ with single exposure is 0.25 , but the generally accepted manufacturability limit is 0.27 . The current industry-standard imaging tool has a wavelength of $193 \mathrm{~nm}$. Future imaging tools are proposed to operate in the extreme ultraviolet (EUV) range with a $\lambda$ of $13.4 \mathrm{~nm}$, however, the EUV technology will most likely not be viable until after 2013. With water immersion lithography, the maximum achievable NA $\sim 1.35$. Increasing the NA requires simultaneous development of a high-index lens material along with high-index fluids and high-index resists. Without major breakthroughs in optical materials, NA will plateau near 1.35. Given these parameters, the current $\mathrm{CD}$ limit is $\sim 38 \mathrm{~nm}$ half-pitch.

*Author to whom all correspondence should be addressed. $1932-5150 / 2009 / \$ 25.00$ @ 2009 SPIE
To enable lithography at sub-38 nm half-pitch, the industry will need to consider alternative resolution enhancement technologies. Two exposure passes have been proposed as a possible resolution enhancement technique for existing photolithography imaging systems. A single mask with high feature density that is difficult to resolve can be split into two exposures, each with lower feature density that can be easily resolved. When combined, the two exposures replicate the original mask.

\subsection{Double-Exposure versus Double-Patterning Lithography}

Double-exposure lithography (DEL) and double-patterning lithography (DPL) are proposed approaches to performing the two-exposure passes. DEL is defined as a twoexposure-pass lithographic process that does not require the removal of the wafer from the exposure tool chuck between passes. DPL is defined as a two-exposure-pass lithographic process that requires a chemical development of the photoresist layers and possibly an intermediate etch step. The DPL processing approaches will require the removal of the wafer from the exposure tool chuck and loss of overlay registration. DEL and DPL processes are illustrated in Fig. 1. The benefits of DEL and DPL principally include the ability to use existing exposure tools to print technology nodes below the NA limit for single-exposure processes. This could mean a lower cost of ownership because these techniques can, in principle, be deployed without a costly capital investment. However, the two-exposure passes require doubling the number of masks and reduced through- 


\section{Double Patterning}

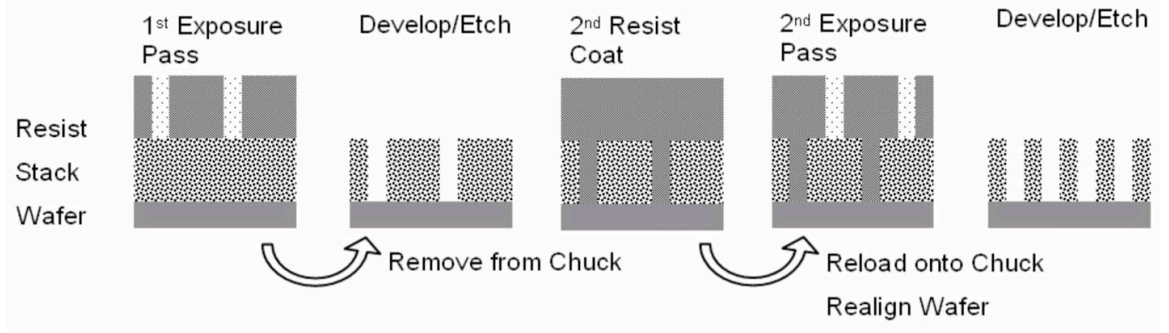

Double Exposure

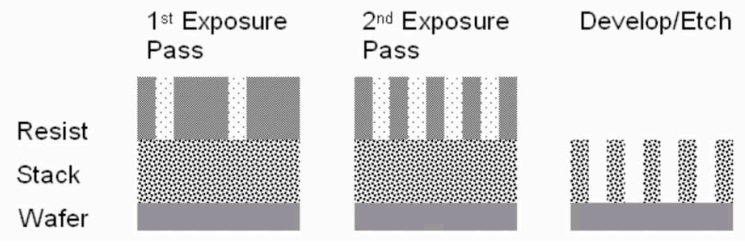

Fig. 1 Comparison of the DPL (development scheming shown) and DEL processes.

put due to increased processing time. The process time is dramatically increased in the DPL process because of the additional process steps compared to the DEL process. In addition, the removal of the wafer from the wafer chuck between exposures poses severe overlay issues that may be difficult to overcome, especially at the CDs, where this technology will be implemented. The DEL process only introduces an additional exposure pass, and because the wafer is not removed from the imaging tool between exposures, the overlay issues are minimized. The reduced cost of ownership of DEL suggests that it would be the preferred technique.

\subsection{Resist "Memory" Effect}

The DEL infrastructure is currently available on existing state of the art exposure tools. However, imaging below a $k_{1}$ value of 0.25 with double exposure is impossible without the development of new materials. Conventional resists have a "memory" effect that prevents proper replication of the mask image. That is, subthreshold exposure in the first

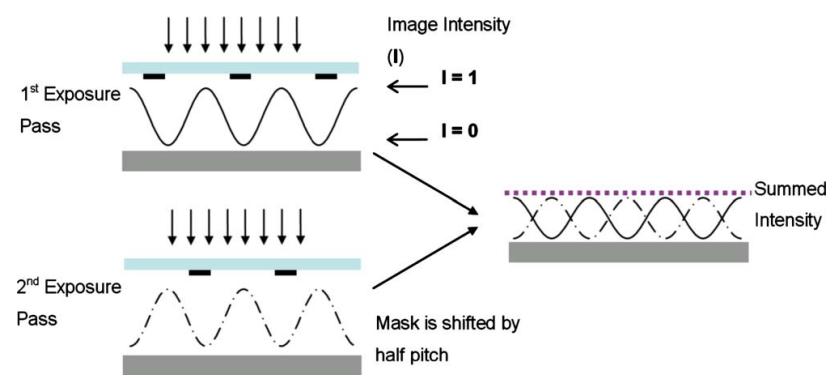

Fig. 2 Summation of the intensity of two-exposure passes and the effect of dose reciprocity. exposure pass reduces the dose required to render the resist soluble in the second exposure pass. For example, the normalized aerial image intensities for the first exposure pass reaching the resist of equal lines and spaces can be described by

$I_{\text {Pass 1 }}=A \cos ^{2}\left(\frac{\pi x}{\text { pitch }}\right)+B$,

where $A$ is a constant describing the amplitude and $B$ is the minimum image intensity. For the second exposure pass, the mask and, consequently, the aerial image are translated by half-pitch and lead to the following intensity function

$$
\begin{aligned}
I_{\text {Pass 2 }} & =A \cos ^{2}\left(\frac{\pi x}{\text { pitch }}+\frac{\pi}{2}\right)+B \\
& =A \sin ^{2}\left(\frac{\pi x}{\text { pitch }}\right)+B .
\end{aligned}
$$

The photochemical response of the resist results in a linear summation of the absorbed intensities from the two exposure pass. This leads to the following intensity function within the resist:

$$
\begin{aligned}
I_{\text {Sum }} & =I_{\text {Pass } 1}+I_{\text {Pass } 2} \\
& =A \cos ^{2}\left(\frac{\pi x}{\text { pitch }}\right)+A \sin ^{2}\left(\frac{\pi x}{\text { pitch }}\right)+2 B \\
& =A+2 B=\text { a constant. }
\end{aligned}
$$

Consequently, the two individual mask images are not resolved when double exposed. This concept is illustrated in Fig. 2. 
The resist system converts the separate light images, intensity versus position, into chemical images, chemical composition versus position. Mathematically, this conversion of the light image into a chemical image can be represented by a translation function $f(I)$. In the case of standard resist systems, this translation function has the linear addition property

$f\left(I_{\text {Pass 1 }}+I_{\text {Pass 2 }}\right)=f\left(I_{\text {Pass 1 }}\right)+f\left(I_{\text {Pass 2 }}\right)$.

Resolving the mask features (i.e., generation of image contrast) requires a material with a nonlinear response to exposure such that

$f\left(I_{\text {Pass 1 }}+I_{\text {Pass 2 }}\right) \neq f\left(I_{\text {Pass 1 }}\right)+f\left(I_{\text {Pass 2 }}\right)$

and the "resist memory" behavior is minimized.

\subsection{Potential DEL Materials}

Several materials have been proposed to implement a nonlinear response to exposure and theoretically permit double-exposure pitch doubling, including contrast enhancement layers (CELs), two-photon materials, intermediate state two-photon (ISTP) materials, and optical threshold layers (OTLs). These materials and their theory of operation are described in the following sections.

\subsubsection{CEL}

CELs are strongly absorbing materials that increase transparency, or photobleach, ${ }^{1}$ when exposed to light. A CEL is normally applied directly on top of the resist layer. During exposure, energy is first devoted to photobleaching the CEL. As the CEL becomes transparent, the energy is then able to reach the resist and initiate the solubility switch. Light can only penetrate through the CEL in regions where aerial image intensities are high (nonopaque regions on the mask) and cannot reach the resist in regions where aerial image intensities are lower (opaque regions on the mask). This introduces a nonlinear transfer of the applied aerial image into the photoresist and improves the resolution. CELs can be divided into two different subtypes, namely, reversible (rCEL) and irreversible (irCEL). The main difference between the two subtypes is that, in rCELs, the photobleached regions can return to the initial opaque state between exposure passes, whereas in irCELs, the photobleaching is irreversible. Details on the existing chemistries and transmission characteristics for CELs have been described in previously published work ${ }^{2-7}$ and are not discussed here.

We have investigated the use of rCELs with 50-nm line/ space patterns in single-exposure mode through simulation with PROLITH 9.3. The film stack from top to bottom consisted of rCEL, $100 \mathrm{~nm}$ of typical 193-nm resist, and $28 \mathrm{~nm}$ of a single layer of bottom antireflective coating (BARC) $(n=1.82, k=0.46)$. The substrate was silicon with a 2-nm layer of $\mathrm{SiO}_{2}$. Briefly, the simulation conditions are listed in Table 1. The results are shown in Figs. 3 and 4

Figures 3 and 4 show that the use of the rCEL moderately increased the depth of focus for the process, but the increase comes at the cost of increases in exposure doses,
Table 1 Simulation parameters used for the $50 \mathrm{~nm}$ L/S with rCEL in single-exposure mode.

\begin{tabular}{|c|c|}
\hline Property & Value \\
\hline NA & 1.2 \\
\hline Illumination & Cross-quasar \\
\hline Blade angle (deg) & 35 \\
\hline$\sigma_{\text {outer }}$ & 0.9 \\
\hline$\sigma_{\text {inner }}$ & 0.7 \\
\hline Resist refractive index & 1.70 \\
\hline Resist Dill $\mathrm{A}\left(\mu m^{-1}\right)$ & 0 \\
\hline Resist Dill B $\left(\mu m^{-1}\right)$ & 1.47 \\
\hline Resist Dill C $\left(\mathrm{cm}^{2} / \mathrm{mJ}\right)$ & 0.0478 \\
\hline Resist $[\mathrm{Q}] /[\mathrm{PAG}]$ & 0.2 \\
\hline Resist $M_{\text {th }}$ & 0.75 \\
\hline Resist $D_{\mathrm{H}}\left(\mathrm{nm}^{2} / \mathrm{s}\right)$ & 0.223 \\
\hline Resist $D_{\mathrm{Q}}\left(\mathrm{nm}^{2} / \mathrm{s}\right)$ & 0.0 \\
\hline Resist $k_{\mathrm{a}}(1 / \mathrm{s})$ & 0.100 \\
\hline Resist $k_{\mathrm{b}}(1 / \mathrm{s})$ & $4.85 \times 10^{8}$ \\
\hline rCEL thickness (nm) & $0-500$ \\
\hline rCEL refractive index & 1.69 \\
\hline rCEL Dill A $\left(\mu \mathrm{m}^{-1}\right)$ & $0-5$ \\
\hline rCEL Dill B $\left(\mu \mathrm{m}^{-1}\right)$ & 0 \\
\hline rCEL Dill C $\left(\mathrm{cm}^{2} / \mathrm{mJ}\right)$ & 0.11 \\
\hline Development model & Enhanced mack model \\
\hline Development $R_{\max }(\mathrm{nm} / \mathrm{s})$ & 400 \\
\hline Development $R_{\min }(\mathrm{nm} / \mathrm{s})$ & 0.01 \\
\hline Development $R_{\text {resin }}(\mathrm{nm} / \mathrm{s})$ & 400 \\
\hline Development $n$ & 3 \\
\hline Development I & 3.98 \\
\hline Development time (s) & 60 \\
\hline
\end{tabular}

almost by a factor of 10 in the case of $A=5.0 \mu \mathrm{m}^{-1}$ when compared to the no-CEL case, and in most cases decreases in exposure latitude (EL).

\subsubsection{Two-photon materials}

Two-photon photoresist systems involve the incorporation of photoacid generators (PAGs) that require the simulta- 


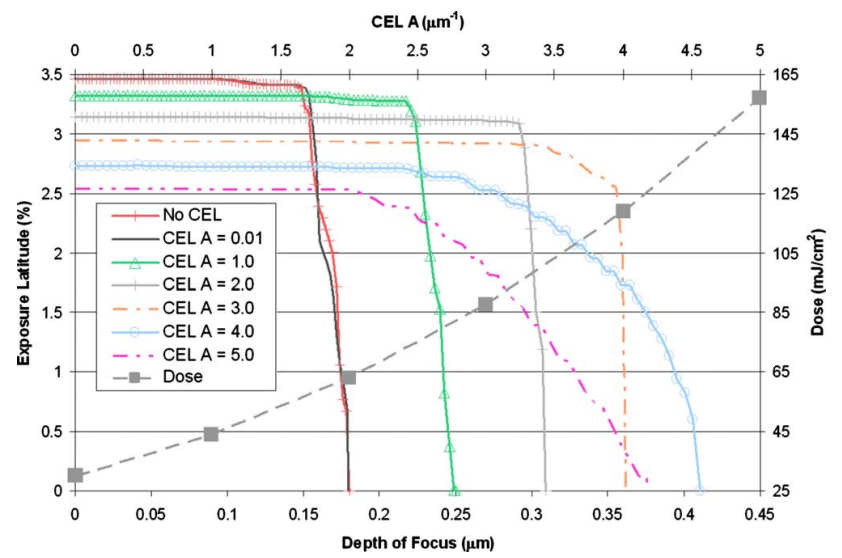

Fig. 3 Process window of 50-nm L/S with $400 \mathrm{~nm}$ of rCEL varying the Dill A parameter.

neous absorption of two photons to induce the photochemical acid generation. The chemical reaction for a two-photon PAG can be described by the following reaction:

$\mathrm{PAG}+I(h \nu)+I(h \nu) \stackrel{\phi}{\longrightarrow}$ acid

where $\phi$ is the quantum efficiency of the two-photon photochemical reaction. Because the simultaneous absorption of two photons is required for the reaction, the probability of conversion is proportional to the light intensity squared, which provides a nonlinear response to exposure energy

$f(I) \approx I^{2}$,

and the DEL conversion is

$$
\begin{aligned}
f\left(I_{\text {Pass 1 }}\right)+f\left(I_{\text {Pass 2 }}\right) & \approx I_{\text {Pass 1 }} I_{\text {Pass 1 }}+I_{\text {Pass } 2} I_{\text {Pass 2 }} \\
& \neq f\left(I_{\text {Pass 1 }}+I_{\text {Pass 2 }}\right) .
\end{aligned}
$$

Unlike the CEL, two-photon materials are not enhancement layers that are applied on top of existing resists, but rather the nonlinear response is incorporated directly into the resist formulation. This eliminates complexities introduced

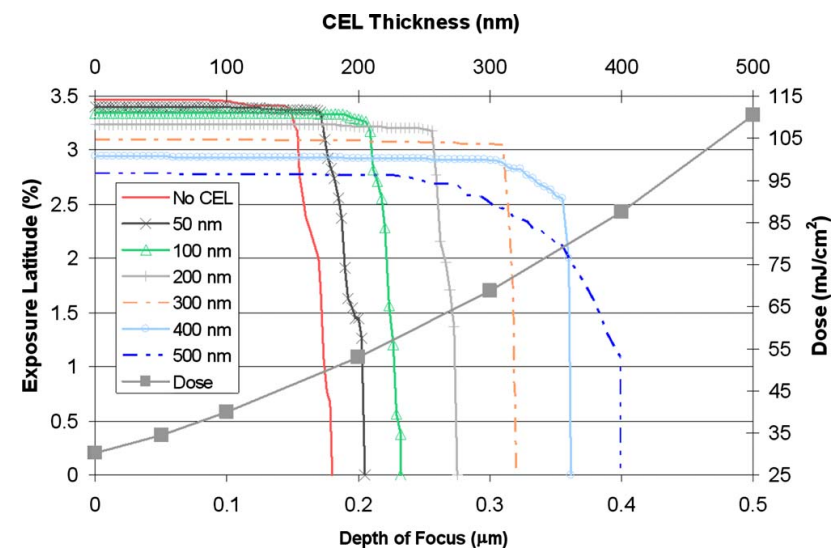

Fig. 4 Process window of $50 \mathrm{~nm} \mathrm{~L} / \mathrm{S}$ with $\mathrm{rCEL}$ having Dill A parameter of $3.0 \mu \mathrm{m}^{-1}$ varying rCEL layer thickness. by the addition of an extra film layer, such as depth of focus and material compatibility.

Two-photon resist systems for microfabrication using laser writing systems have been reported previously. ${ }^{8}$ These systems employ specially designed PAGs with high twophoton absorbance cross sections. High-efficiency twophoton PAGs have not yet been developed to work with $193 \mathrm{~nm}$. Analysis of the two-photon reaction kinetics suggests that a very large increase in exposure source output would be required to produce a viable level of process throughput.

For example, the two-photon absorbance transition probability of a two-photon PAG can be more specifically described by

$P_{2 h \nu} \approx \sigma_{2} \int I^{2} d t$

where $\sigma_{2}$ is the two-photon absorbance cross section, with a typical value of approximately $5 \times 10^{-47} \mathrm{~s} \mathrm{~cm}^{4} /$ photon (Ref. 9) for current two-photon PAGs. Using characteristics typical of current exposure tools $\left(193 \mathrm{~nm}, 10 \mathrm{~mW} / \mathrm{cm}^{2}\right.$, $4000 \mathrm{~Hz}, 20 \mathrm{~ns}$ FWHM) and assuming a Gaussian intensity profile, the absorbance transition probability per laser pulse is

$$
\begin{aligned}
P_{2 h \nu} & =\frac{\sigma_{2}}{2}\left(\frac{A 0 \lambda}{h c}\right)^{2} \frac{0.664}{\mathrm{FWHM}} \\
& =\sigma_{2} 9.77 \times 10^{31} \text { photon }^{2} / \text { pulse s cm }^{4},
\end{aligned}
$$

where $A 0$ is the energy per unit area delivered by each pulse, $\lambda$ is the exposure wavelength, $h$ is Planck's constant, $c$ is the speed of light, and FWHM is the full width at half-maximum of the laser pulse. ${ }^{10}$ Finally, the number of laser pulses necessary to produce $20 \%$ conversion of PAG into acid, assuming a quantum efficiency of 1.0 for the $\mathrm{PAG}$, is

Number of pulses

$$
\begin{aligned}
& 0.20
\end{aligned}
$$

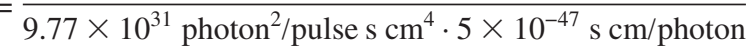

$$
\begin{aligned}
& \approx 4 \times 10^{13} \text { pulses. }
\end{aligned}
$$

Alternatively stated, the fraction of converted PAG for a 10 -s exposure would be on the order of

$$
\begin{aligned}
P_{2 h \nu} & =4.9 \times 10^{-15} \mathrm{pulse}^{-1} \cdot 10 \mathrm{~s} \times 4000 \mathrm{pulses} / \mathrm{s} \\
& \approx 2 \times 10^{-10} .
\end{aligned}
$$

Given the reaction kinetics of two-photon reactions and the typical exposure source intensity of the projection photolithographic processes, the analysis described in Eqs. (10)-(13) indicates that a two-photon photoresist system would require very long exposure time to achieve sufficient amount of photochemical reaction for imaging. The probability in Eq. (11) can be increased by reducing the FWHM of the laser, i.e. using a femtosecond laser, increasing $A 0$ to similar that of a direct laser write focused beam, or increasing $\sigma_{2}$ by nine orders of magnitude to 5 $\times 10^{-38} \mathrm{~s} \mathrm{~cm}^{4}$ / photon. Therefore, without a very large increase in exposure source intensity, the existing two-photon 
PAG materials could not maintain the throughput level typical of current lithographic process. As such, the twophoton material type was not included in the simulation studies.

\subsubsection{ISTP materials}

ISTP layers are materials that generate acid molecules in a two-step process. Similar to two-photon materials, ISTP materials alter the acid-generation behavior of the resist medium. Although each step requires the absorption of a photon, ISTP materials are not true two-photon processes in that the acid production does not have a quadratic dependence on dose. The reaction sequence is

$$
\begin{gathered}
A+I(h \nu) \underset{1 / \tau_{1}}{\stackrel{\sigma_{1}}{\rightleftharpoons}} B \\
B+I(h \nu) \underset{1 / \tau_{2}}{\stackrel{\sigma_{2}}{\rightleftharpoons}}[\text { acid }],
\end{gathered}
$$

where $\sigma_{1}$ and $\sigma_{2}$ represent the cross sections with respective quantum efficiencies included and $\tau_{1}$ and $\tau_{2}$ represent the lifetimes of the species. The system of rate equations governing the production of acid is as follows:

$$
\begin{aligned}
& \frac{d[A]}{d t}=-\sigma_{1} I(h \nu)[A]+\frac{1}{\tau_{1}}[B] \\
& \frac{d[B]}{d t}=\sigma_{1} I(h \nu)[A]-\left(\frac{1}{\tau_{1}}+\sigma_{2} I(h \nu)\right)[B]+\frac{1}{\tau_{2}}[\text { acid }] \\
& \frac{d[\text { acid }]}{d t}=\sigma_{2} I(h \nu)[B]-\frac{1}{\tau_{2}}[\text { acid }] .
\end{aligned}
$$

For cases where

$$
\frac{1}{\tau_{1}} \gg \sigma_{1} I(h \nu) \text { and } \frac{1}{\tau_{2}} \approx 0,
$$

the steady-state approximation may be applied such that

$$
\frac{d[B]}{d t}=0 .
$$

After integration and rearrangement, the [acid] has the following dependence on dose during exposure

$$
\frac{[\mathrm{acid}]}{[A]}=\frac{\sigma_{1} \sigma_{2}\left(\int_{\text {pulse }} I d t\right)^{2}}{t_{f} / \tau_{1}+\sigma_{2} \int_{\text {pulse }} I d t},
$$

where $t_{f}$ is the pulse cycle time (inverse of the laser repetition rate) and the integral represents the dose delivered. An examination of Eq. (18) reveals that higher reaction orders between 1.0 and 2.0 can be achieved if the parameters in the denominator are tuned such that the $t_{f} / \tau_{1}$ term dominates over the dose-dependent term. Although ISTP materials do not exhibit true two-photon behavior, they may require significantly lower doses to generate acid compared to two-photon resists. The trade-off between lower reaction orders may be offset by the lower doses.
The behavior of ISTP materials depends on the ability of the intermediate species to revert to the initial state or possibly decay to another species. A buildup of the intermediate species will effectively render the sequence to become a first-order reaction that is controlled by a rate-limiting step. Therefore, the characteristics of the exposing laser, such as the energy per unit area delivered by each pulse $(A 0)$, pulse cycle time $\left(t_{f}\right)$, and FWHM also have to be considered.

\subsubsection{Optical threshold materials}

OTLs are materials that require the absorption of a threshold exposure dose to induce a photochemical event. The exposure threshold gives the material a region of nonlinear response to exposure dose and allows OTLs to be used as double-exposure resists. Nonlinearity derives from the fact that any dose absorbed below the threshold does not cause reactions to occur. On reaching the threshold dose, the threshold photochemical response occurs. To prevent a quencherlike response that does not provide the necessary nonlinearity behavior for DEL (only provides a dose offset), the threshold response is not further impacted by additional dose above the threshold dose. The ideal binary step change behavior is more clearly described by the following simple piecewise function:

$f(I)=\left\{\begin{array}{cc}\text { Threshold response, } & E_{\text {actual }} \geqslant E_{\text {th }} \\ \text { No response, } & E_{\text {actual }}<E_{\text {th }}\end{array}\right.$

where

$E_{\text {actual }}=\int_{\text {pulses }} I \cdot d t$,

$E_{\text {actual }}$ and $E_{\text {th }}$ represent the actual dose received by the material and the threshold dose, respectively.

Analogous thermal resist systems are already in use in the printing industry. ${ }^{11}$ Thermal resists rely on a thermal image instead of an optical image. The thermal image is derived from the absorbance of high-intensity light images. Thermal resists use the absorbed thermal energy to induce a phase change in the system and cause the solubility switch as opposed to traditional chemical resists, which use the energy to carry out a chemical reaction. Phase changes are ideal as the dose-dependent mechanisms in OTL materials because the threshold response is inherently built into the thermodynamics of the system. For example, in the solidto-liquid transition of water, the thermal dose required to melt the ice is the sum of the energy required to bring the water from the initial temperature to the melting temperature and the latent heat of fusion. Below the threshold dose, the transition does not occur. The system also does not have any memory effects to the thermal dose as the thermal dose required for melting per unit mass at a given initial temperature remains constant even after multiple heating and cooling cycles.

Chapman et al. have investigated inorganic thermal resist systems ${ }^{12}$ that use $\mathrm{Bi} / \mathrm{In}$ bilayers as an etch masking layer for silicon. However, the use of $\mathrm{Bi}$ and $\mathrm{In}$ metals are not compatible with the photolithography process because the target semiconductor devices are very susceptible to metal contamination. Chemical systems with similar prop- 
Lee et al.: Materials modeling and development for use in double exposure...

Table 2 Parameters used for the based resist system.

\begin{tabular}{|c|c|}
\hline Property & Value \\
\hline Resist refractive index, $n$ & 1.70 \\
\hline Resit Dill $\mathrm{A}\left(\mu \mathrm{m}^{-1}\right)$ & 0 \\
\hline Resist Dill B $\left(\mu \mathrm{m}^{-1}\right)$ & 1.47 \\
\hline Resist Dill C $\left(\mathrm{cm}^{2} / \mathrm{mJ}\right)$ & 0.0478 \\
\hline $\begin{array}{l}\text { Relative quencher concentration, } \\
{[[\mathrm{Q}] /[\mathrm{PAG}]}\end{array}$ & 0.2 \\
\hline Development model & Original mack mode \\
\hline Development $R_{\max }(\mathrm{nm} / \mathrm{s})$ & 100 \\
\hline Development $R_{\min }(\mathrm{nm} / \mathrm{s})$ & 0.1 \\
\hline Threshold inhibitor concentration, $M_{\text {th }}$ & 0.75 \\
\hline Dissolution selectivity parameter & 25.0 \\
\hline Acid diffusivity, $D_{\mathrm{H}}\left(\mathrm{nm}^{2} / \mathrm{s}\right)$ & 0.223 \\
\hline Quencher diffusivity, $D_{\mathrm{Q}}\left(\mathrm{nm}^{2} / \mathrm{s}\right)$ & 0.0 \\
\hline $\begin{array}{l}\text { Amplification reaction rate constant, } k_{\mathrm{a}} \\
\qquad(1 / \mathrm{s})\end{array}$ & 0.100 \\
\hline $\begin{array}{l}\text { Acid base quenching rate constant, } k_{\mathrm{b}} \\
\qquad(1 / \mathrm{s})\end{array}$ & $4.85 \times 10^{8}$ \\
\hline Development time (s) & 20 \\
\hline
\end{tabular}

erties for optical images have to be developed to use this technology with lithographic imaging systems.

Analogous chemical systems will most likely also need to use the optical image to induce a phase change in the material, which will then allow a solubility switch. The phase change will replicate the behavior of thermal resists in that the absorption of a threshold dose is required to induce phase change. Below the threshold, no solubility switch should occur and the material should not have resist memory.

\subsection{Feasibility Studies}

These materials are not the only possibilities, but they do provide a reasonable range of resist designs to explore the feasibility of DEL as a technology choice. Of the proposed materials, only CELs and two-photon materials have been extensively studied and have established chemical systems. ISTP and OTL materials do not currently exist for use in semiconductor applications. However, their theoretical mechanisms are considered to test their viability as possible DEL candidates. This work investigates the feasibility of the materials for use in DEL applications through simulation and is meant to guide our materials development effort.

\section{Simulation Conditions}

The performance of the different material types in doubleexposure mode and the dependence on their material prop-
Table 3 Simulation parameters used for the reversible CEL material.

\begin{tabular}{cc}
\hline \hline Parameter & Value \\
\hline rCEL refractive index, $n$ & 1.69 \\
rCEL Dill A $\left(\mu \mathrm{m}^{-1}\right)$ & $0-50$ \\
rCEL Dill B $\left(\mu \mathrm{m}^{-1}\right)$ & 0 \\
rCEL Dill C $\left(\mathrm{cm}^{2} / \mathrm{mJ}\right)$ & 0.11 \\
\hline \hline
\end{tabular}

erties were evaluated by computer simulation using a combination of the commercially available PROLITH V9.3 lithography simulator from KLA-Tencor and custom code. In all cases, the optical imaging portion was performed with PROLITH. The material responses of the reversible CEL was studied using the PROLITH simulator. However, commercial models for the ISTP and OTL materials do not yet exist because the materials are not currently used in production; thus a custom simulator was developed to model the material behaviors in these systems.

\subsection{Imaging Setup}

A half-pitch CD of $25 \mathrm{~nm}$ was targeted using a 1.2-NA water-immersion exposure system in double-exposure mode. This is an effective $k_{1}$ of 0.155 . An azimuthally polarized cross-quadrupole with $\sigma_{\text {center }}=0.8$ and $\sigma_{\text {radius }}=0.15$ was used as the illuminator. Different masks were used for each of the two-exposure passes. The masks were $50-\mathrm{nm}$ line/space phase shift masks with $6 \%$ transmission. The two masks were offset by $50 \mathrm{~nm}$ between exposure passes. As described previously, ${ }^{2}$ DEL with positive tone resists is a trench-based process as opposed to the line-based process expected with a single-exposure pass. Consequently, the target line is expected to form at the interface of the opaque and bright regions as opposed to the center of the bright regions. A focus-exposure matrix was run for each material system, and the resulting CD was observed.

Table 4 Simulation parameters used for the ISTP material.

\begin{tabular}{cc}
\hline \hline Parameter & Value \\
\hline$\sigma_{1}\left(\mathrm{~cm}^{2} / \mathrm{mJ}\right)$ & 0.235 \\
$\sigma_{2}\left(\mathrm{~cm}^{2} / \mathrm{mJ}\right)$ & 0.235 \\
$\tau_{1}(\mathrm{~s})$ & 0.006 \\
$t_{f}$ & 0.00025 \\
$A 0\left(\mathrm{~mJ} / \mathrm{cm}^{2}\right)$ & 0.05 \\
$\phi$ & 1 \\
$\mathrm{FWHM}(\mathrm{ns})$ & 20 \\
\hline \hline
\end{tabular}




\section{$A=0 \mu \mathrm{m}^{-1}$}

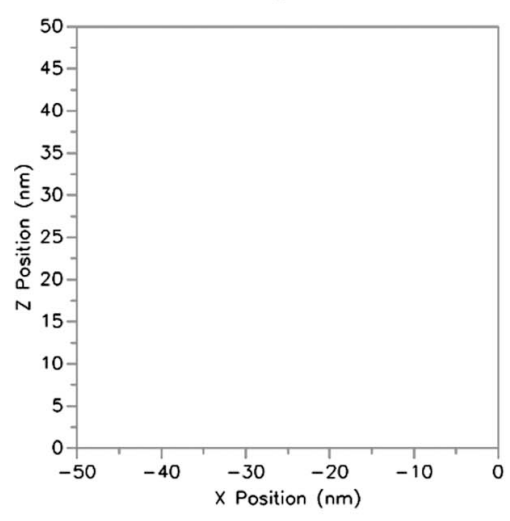

$A=30 \mu \mathrm{m}^{-1}$

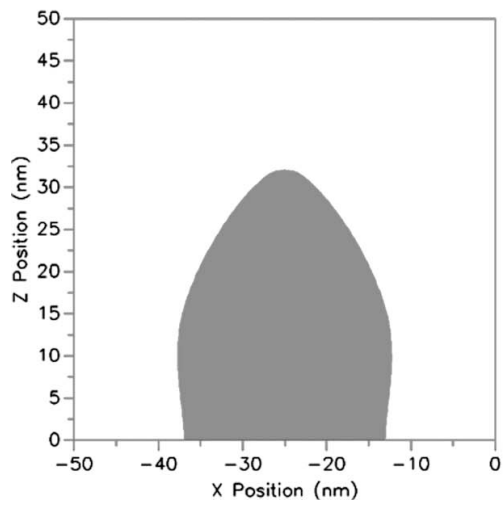

$A=10 \mu \mathrm{m}^{-1}$

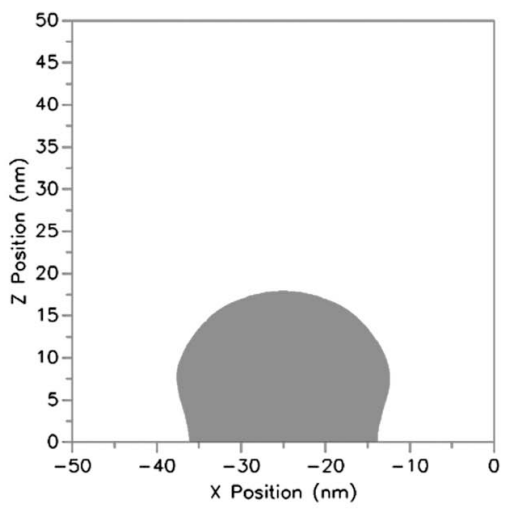

$A=40 \mu \mathrm{m}^{-1}$

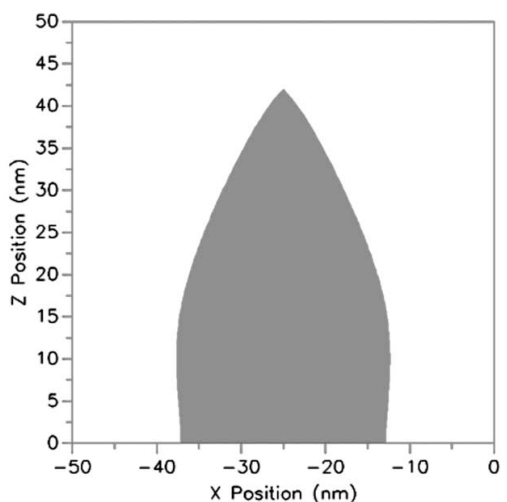

$A=20 \mu \mathrm{m}^{-1}$

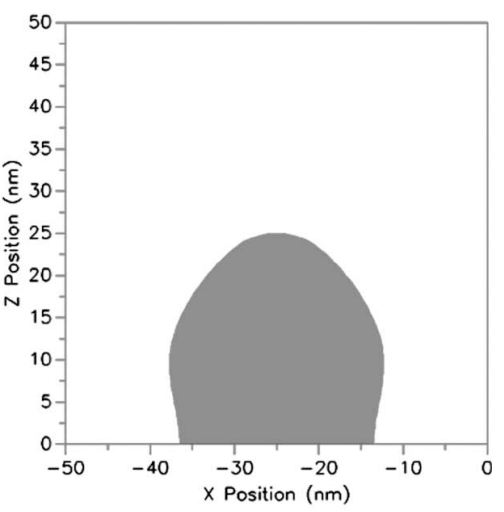

$A=50 \mu \mathrm{m}^{-1}$

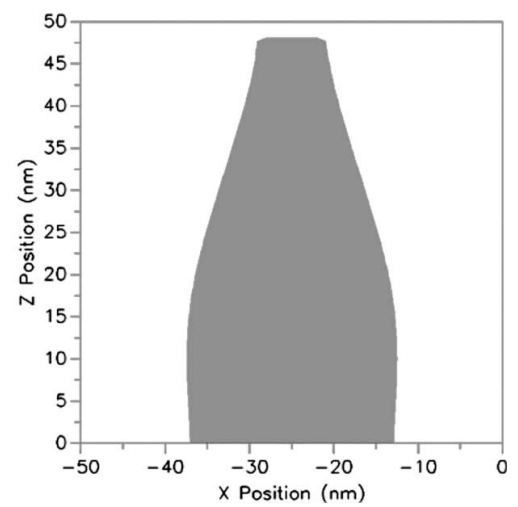

Fig. 5 Resist profile of rCEL with varying Dill A parameter.

\subsection{Film Stack and Base Resist System}

The film stack consisted of $50 \mathrm{~nm}$ of resist on $31 \mathrm{~nm}$ of a single layer of BARC $(n=1.82, k=0.46)$. The substrate was silicon with a 2-nm layer of $\mathrm{SiO}_{2}$. The resist simulations were based on a typical 193-nm resist system. The base resist parameters are shown in Table 2. In the case of the OTL and ISTP materials, the acid generation behavior differs from the base resist and was described with custom models.

\subsection{Reversible CEL Simulation Parameters}

To study the CEL behavior, a 50-nm CEL was applied on top of the film stack. The Dill A parameter was varied from 10 to $50 \mu \mathrm{m}^{-1}$. The CEL parameters are shown in Table 3. Because a reversible CEL system provides better performance than an irreversible system, the rCEL was selected for the study.

\subsection{ISTP Simulation Parameters}

To study the performance of the ISTP material, the acidgeneration behavior described in Section 2.3 was solved using a fourth-order Runge-Kutta method. ${ }^{13}$ The parameters and values used in the simulation are listed in Table 4. To simplify the calculations, $\tau_{2}$ was assumed to be very large such that the conversion of the intermediate species to acid is assumed to be irreversible.

\subsection{OTL Simulation Parameters}

The behavior of an ideal OTL material is shown in Eq. (19). However, because OTL materials are not yet available, their mechanisms were also not yet implemented in PROLITH V9.3. Therefore, it was necessary to devise a method to capture the OTL phenomenon within the PROLITH framework. In this case, acid-generation behavior

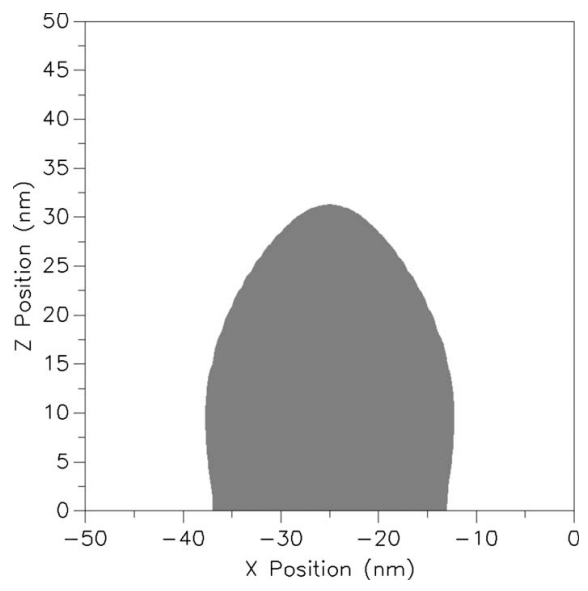

Fig. 6 Resist profile for ISTP material. 


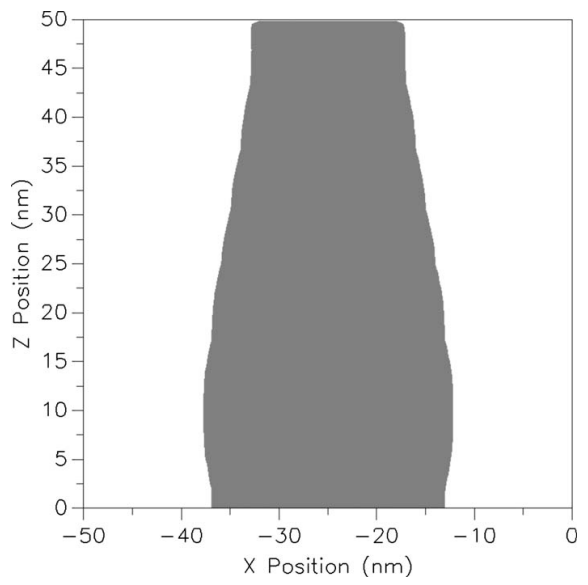

Fig. 7 Resist profile for OTL material.

was selected to be the threshold response, although it is important to note that the eventual mechanism is not limited to and may not necessitate acid generation. Equation (19) was modified to describe the threshold response in conversion in the following manner:

$f(I)=\left\{\begin{array}{cl}{[\mathrm{PAC}]_{\mathrm{th}},} & E_{\text {actual }} \geqslant E_{\mathrm{th}} \\ 1, & E_{\text {actual }}<E_{\mathrm{th}}\end{array}\right.$

where

$E_{\text {actual }}=\int_{\text {pulses }} I d t$,

as before, $E_{\text {actual }}$ and $E_{\text {th }}$ represent the actual dose received by the material and the threshold dose, respectively. $[\mathrm{PAC}]_{\mathrm{th}}$ is the stepwise conversion concentration of the photoactive compound after reaching $E_{\mathrm{th}}$. A dose threshold, $E_{\mathrm{th}}$, of $10 \mathrm{~mJ} / \mathrm{cm}^{2}$ was used with a threshold photoacid compound conversion, $[\mathrm{PAC}]_{\mathrm{th}}$, value of 0.75 .
Table 5 Summary of resist profile metrology.

\begin{tabular}{cccc}
\hline Material & $\begin{array}{c}\text { Dose } \\
\left(\mathrm{mJ} / \mathrm{cm}^{2}\right)\end{array}$ & $\begin{array}{c}\text { Sidewall angle } \\
(\mathrm{deg})\end{array}$ & $\begin{array}{c}\text { Resist loss } \\
(\mathrm{nm})\end{array}$ \\
\hline $\operatorname{rCEL} A=10 \mu \mathrm{m}^{-1}$ & 25 & 75 & 32 \\
$\operatorname{rCEL} A=20 \mu \mathrm{m}^{-1}$ & 38 & 74 & 25 \\
$\operatorname{rCEL} A=30 \mu \mathrm{m}^{-1}$ & 56 & 71 & 18 \\
$\operatorname{rCEL} A=40 \mu \mathrm{m}^{-1}$ & 80 & 70 & 8.1 \\
$\operatorname{rCEL~} A=50 \mu \mathrm{m}^{-1}$ & 110 & 75 & 2.0 \\
ISTP & 37 & 72 & 19 \\
OTL & 39 & 82 & 0.18 \\
\hline \hline
\end{tabular}

\section{Results and Discussion}

All three materials yielded nonlinear resist response when used in double-exposure mode. The resist profiles are shown in Figs. 5-7 and summarized in Table 5.

The simulation results from the focus-exposure experiments were analyzed using commercially available ProDATA V1.4.3 from KLA-Tencor to generate simulated Bossung plots and EL versus depth of focus (DOF) plots. The results are shown in Figures 8-10 and are summarized in Table 6. Simulated EL versus DOF plots with respect to the different Dill A parameters are shown in Fig. 11.

It is important to note that CD was the only output metric considered in the process window calculations. In most manufacturing environments, other parameters such as side-wall angle and resist loss would also need to be optimized to produce functional devices. However, the main goal of this work is to demonstrate the proof of concept of the theoretical materials. Optimization of such parameters is beyond the scope of this work.

Figure 5 shows the effects of the Dill A parameter on the resist profile. For the test case of a Dill A parameter of $0 \mu \mathrm{m}^{-1}$, no resist profile was observed. This finding is con-
Critical Dimension vs. Focus/Exposure for rCELA $=20$ um $^{\wedge}-1$

$C D$

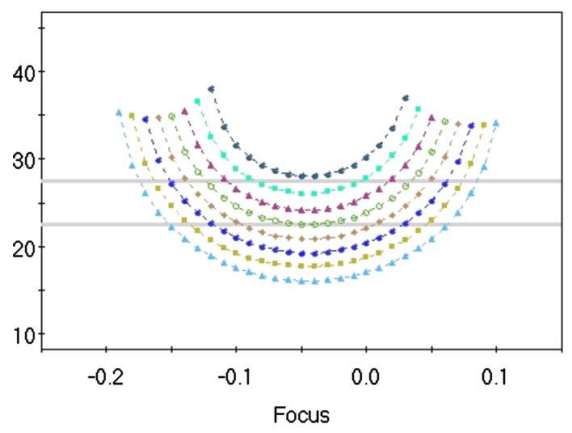

Exposure Latitude vs. DOF for rCELA $=20 \mathrm{um}^{\wedge}-1$

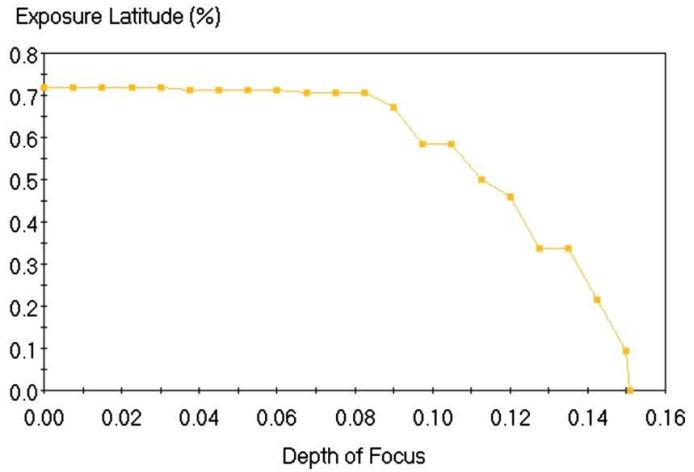

Fig. 8 Simulated Bossung plot and EL versus DOF for rCEL material. 
Critical Dimension ys. Focus/Exposure

for ISTPA0 $=0.05 \mathrm{~mJ} / \mathrm{cm}^{\wedge} 2$. Tau $1=0.006 \mathrm{~s}$

$\mathrm{CD}$

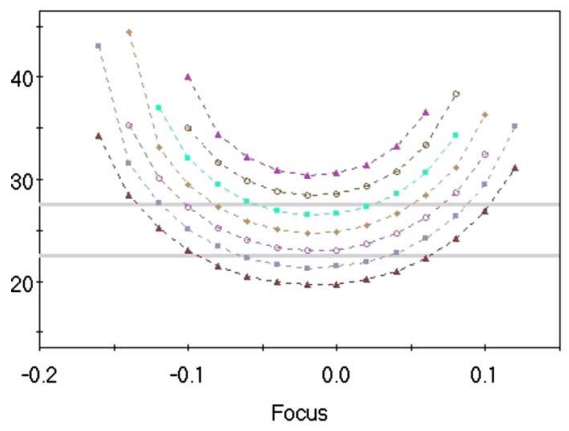

Exposure Latitude vs. DOF for ISTPAO $=0.05 \mathrm{~mJ} / \mathrm{cm}^{\wedge} 2$. Tau $1=0.006 \mathrm{~s}$

Exposure Latitude (\%)

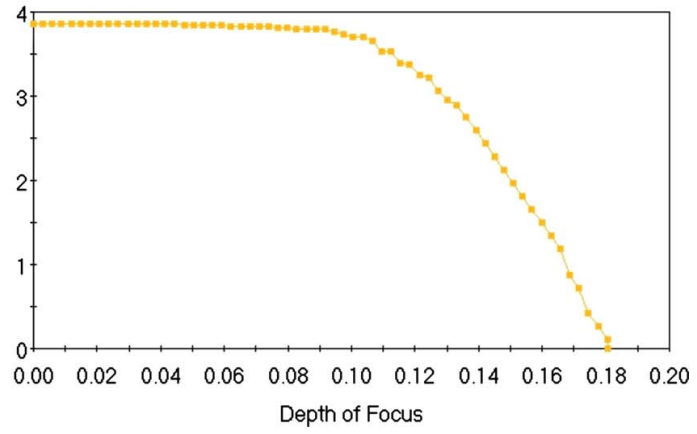

Fig. 9 Simulated Bossung plot and EL versus DOF for ISTP material.

sistent with the behavior of conventional resists because, with a Dill A parameter of $0 \mu \mathrm{m}^{-1}$, the effect of the CEL disappears.

In all cases, increasing the Dill A parameter decreased resist loss and improved the shape of the resulting image. Figure 11 shows that the process window was also widened with the increase. Increasing the Dill A parameter showed improvement for EL. However, only marginal improvement was observed for the DOF. Increasing the Dill A parameter also led to increases in the dose requirement. An increase in the Dill A parameter from 10 to $50 \mu \mathrm{m}^{-1}$ required an 4.4-fold increase in dose. Another factor to consider is the feasibility of obtaining rCEL materials with high Dill A values. Without increasing the Dill A parameter, it is theoretically possible to increase the absorbance of the rCEL layer by increasing the layer thickness. However, the oblique incident angles resulting from operating at NA values of $>1$ may lead to loss in DOF if the layer becomes too thick. rCEL materials showed nonlinear behavior in DEL mode; however, image quality and process window improvement was only observed for rCELs with very high Dill A parameters $\left(>30 \mu \mathrm{m}^{-1}\right)$. Even if physical analogs with such high Dill A parameters are obtainable, the improvements are marginal and come at the cost of large dose increases.

Table 5 shows that an rCEL with Dill A parameter of $20 \mu^{-1}$ has a comparable dose requirement to that of ISTP and OTL materials. Results from this run were used for subsequent comparisons with ISTP and OTL materials.

Figure 6 shows the resist profile of the ISTP material. The profile is comparable to rCEL having slightly lower sidewall angle, $72 \mathrm{deg}$, and reduced resist loss, $19 \mathrm{~nm}$. Table 6 shows that ISTP has a larger process window than rCEL. The parameters of interest affecting the nonlinear acid-generation behavior of the material are the energy per unit area delivered by each pulse, $A 0$, and the reversible rate constant of the intermediate state, $1 / \tau_{1}$. For a given set of laser parameters, large values of $A 0$ or $\tau_{1}$ lead to faster conversion of PAC thus reducing the required exposure dose. However, the dose reduction also leads to a decrease in nonlinearity. Because the laser can only deliver integer numbers of pulses, the magnitude of $A 0$ has to be within a manageable increment such that small deviations in the
Critical Dimension ys. Focus/Exposure for OTL Eth $=10 \mathrm{~mJ} / \mathrm{cm}^{\wedge} 2$. PACth $=0.75$

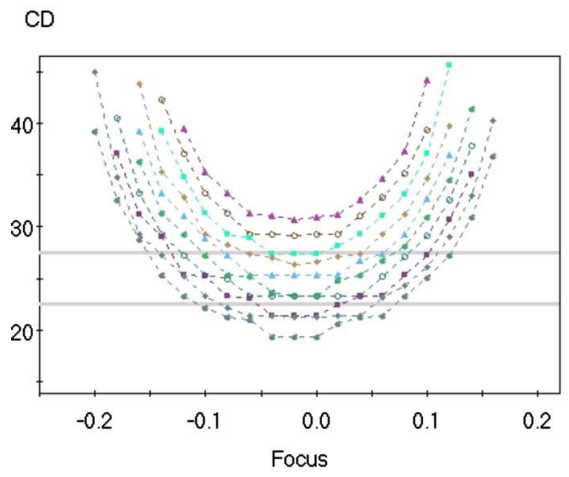

Exposure Latitude vs. DOF for OTL Eth $=10 \mathrm{~mJ} / \mathrm{cm}^{\wedge} 2$. PACth $=0.75$

Exposure Latitude (\%)

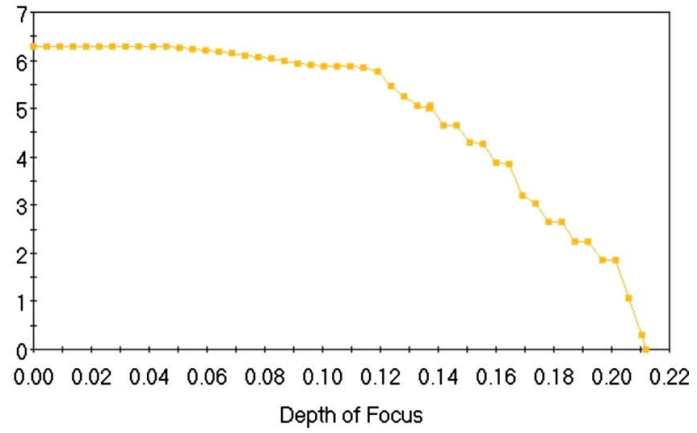

Fig. 10 Simulated Bossung plot and EL versus DOF for OTL material. 


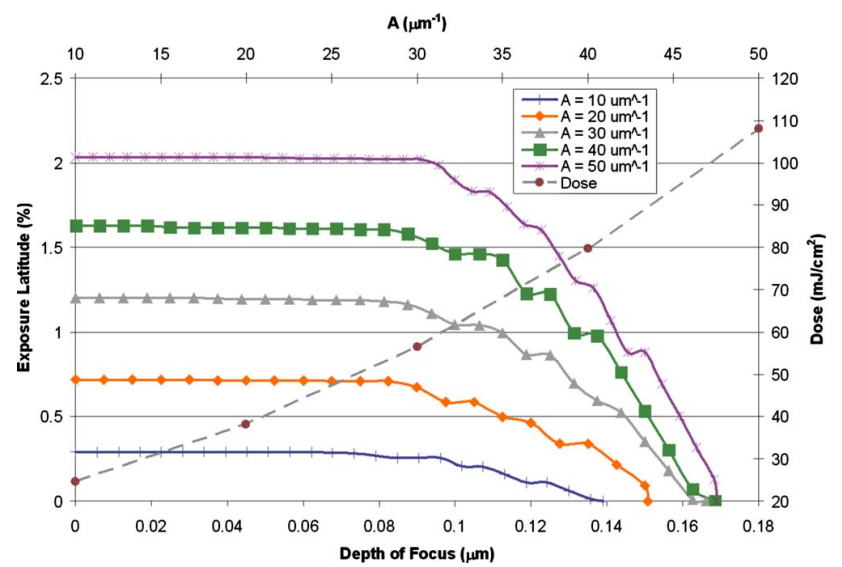

Fig. 11 Process windows of $\mathrm{rCEL}$ materials varying the Dill $A$ parameter.

pulse delivery will not drastically affect the CD. The parameters had to be optimized so that the system will retain nonlinear behavior but at the same time yield features within reasonable exposure dose ranges.

Figure 12 shows the effects of varying $A 0$ and $\tau_{1}$ on the PAC conversion after a target dose of $30 \mathrm{~mJ} / \mathrm{cm}^{2}$ at a relative intensity of 0.3 was delivered. As expected, increases in $A 0$ or $\tau_{1}$ both resulted in faster conversion of [PAC]. For the simulation setup, a [PAC] of 0.8 is desired. Figure 12 shows that this is achievable with all of the $A 0$ values from 0.01 to $0.05 \mathrm{~mJ} / \mathrm{cm}^{2} /$ pulse. However, $A 0$ values of $>0.1 \mathrm{~mJ} / \mathrm{cm}^{2} /$ pulse lead to difficulties in dose deviation management and $\tau_{1}$ values for reasonable chemical systems have an upper limit in the millisecond range. Consequently, $A 0$ and $\tau_{1}$ values of $0.05 \mathrm{~mJ} / \mathrm{cm}^{2}$ and $0.006 \mathrm{~s}$ were selected, respectively. ISTP materials showed a larger process window and improved resist profile than rCEL, and could be a potential DEL material provided that materials with the specified kinetics and time constants can be identified.

Figure 7 shows the resist profile of the OTL material. The profile shows a significant reduction in resist loss com-

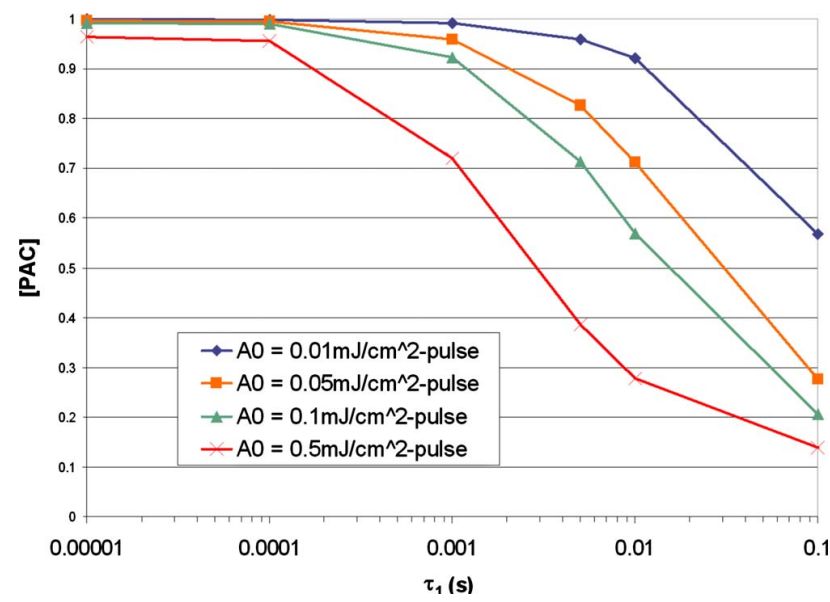

Fig. 12 Effect of $\mathrm{AO}$ and $\tau_{1}$ on the PAC conversion after a target dose of $30 \mathrm{~mJ} / \mathrm{cm}^{2}$ assuming pulse delivery rate at $30 \%$ relative intensity of $\mathrm{A} 0$.
Table 6 Summary of the process windows for the rCEL, ISTP, and OTL materials.

\begin{tabular}{ccc}
\hline \hline Material & DOF $(\mu \mathrm{m})$ & EL $(\%)$ \\
\hline rCEL & 0.11 & 0.58 \\
ISTP & 0.12 & 3.2 \\
OTL & 0.14 & 5.1 \\
\hline \hline
\end{tabular}

pared to both rCEL and ISTP resist profiles and slight improvement in the sidewall angle. The OTL material also has the largest process window of the three materials investigated. The threshold dose requirement behavior of the OTL material served effectively to filter out regions of low intensity. In addition, the threshold conversion response of the PAC resulted in improved image contrast. Because no such physical systems exist, the threshold dose, $E_{\mathrm{th}}$, and PAC conversion, $[\mathrm{PAC}]_{\mathrm{th}}$, were chosen such that they would provide a defined solubility switch within comparable dose ranges. Hypothetical OTL materials showed the best performance (i.e., largest process window and best resist profile) compared to rCEL and ISTP materials and could enable DEL. This conclusion serves as motivation for research directed toward development of such materials.

\section{Conclusions}

DEL offers several advantages over DPL, but it requires new materials with nonlinear dose response. We have employed simulations to explore several potential DEL material options. The modeling results show that two-photon materials will not be feasible unless achievable laser peak power in exposure tools can be significantly increased. rCEL materials demonstrated nonlinear behavior in DEL mode; however, image quality and process window improvement was only observed for rCELs with very high Dill A parameters $\left(>30 \mu \mathrm{m}^{-1}\right)$. Even if physical analogs with such high Dill A parameters are obtainable, the improvements are marginal. ISTP materials showed a larger process window than rCEL. The challenges with this approach are identifying materials with the specified kinetics and the ability to tune the time constants. OTL materials showed the best performance with the largest process window and best resist profile. There are no physically functional optical analogs with the thresholding behavior. Potential mechanisms, either chemical or physical, need to be explored. From our feasibility studies, we believe that the hypothetical ISTP and OTL materials have the greatest potential for use in DEL applications and warrant our investment in materials development.

\section{Acknowledgments}

The authors thank SEMATECH for financial support of this project, Bryan Rice of SEMATECH, KLA-Tencor for the donations of PROLITH and ProDATA licenses, Mark Smith and Trey Graves of KLA-Tencor, Chris Mack, and Intel Corporation for the donations of simulation machines. 
SEMATECH, and the SEMATECH logo are registered servicemarks of SEMATECH, Inc. All other servicemarks and trademarks are the property of their respective owners.

\section{References}

1. B. F. Griffing and P. R. West, "Contrast enhanced lithography," Solid State Technol. 28, 152-157 (1985).

2. J. Byers, S. Lee, K. Jen, P. Zimmerman, N. J. Turro, and C. G. Willson, "Double exposure materials: simulation study of feasibility," J. Photopolym. Sci. Technol. 20, 707-717 (2007)

3. M. M. Krayushkin, B. M. Uzhinov, A. Y. Martynkin, D. L. Dzhavadov, M. A. Kalik, V. L. Ivanov, F. M. Stoyanovich, L. D. Uzhinova, and O. Y. Zolotarskaya, "Thermally irreversible photochromic dithienylethenes," Int. J. Photoenergy 1, 183-190 (1999).

4. R. S. Becker and J. Michl, "Photochromism of synthetic and naturally occurring 2H-chromenes and 2H-pyrans," J. Am. Chem. Soc. 88 5931-5933 (1966).

5. B. D. Grant, N. J. Clecak, R. J. Twieg, and C. G. Willson, "Deep UV photoresists I. Meldrum's diazo sensitizer," IEEE Trans. Electron Devices 28, 1300-1305 (1981)

6. P. R. West, G. C. Davis, and B. F. Griffing, "Contrast enhanced photolithography: application of photobleaching process in microlithography," J. Imaging Sci. 30, 65-68 (1986).

7. E. F. Fleet, S. Gonen, G. D. Cooper, and Z. Chen, "Programmable photolithographic mask and reversible photo-bleachable materials based on nano-sized semiconductor particles and their applications," PCT Int. Appl. WO2004053938 (2004).

8. S. M. Kuebler, K. L. Braun, W. Zhou, J. K. Cammack, T. Yu, C. K. Ober, S. R. Marder, and J. W. Perry, "Design and application of high-sensitivity two-photon initiators for three-dimensional microfabrication," J. Photochem. Photobiol., A 158, 163-170 (2003).

9. S. Zheng, A. Leclercq, J. Fu, L. Beverina, L. A. Padilha, E. Zojer, K Schmidt, S. Barlow, J. Luo, S. H. Jiang, A. K. Y. Jen, Y. Yi, Z. Shuai, E. W. VanStryland, D. J. Hagan, J. L. Bredas, and S. R. Marder, "Two-photon absorption in quadrupolar bis(acceptor)-terminated chromophores with electron-rich bis(heterocycle)vinylene bridges," Chem. Mater. 19, 432-442 (2007).

10. B. Larijani, R. Woscholski, and C. A. Rosser, Chemical Biology: Applications and Techniques, pp. 172-174, Wiley, Chichester, England (2006).

11. D. Gelbart and V. A. Karasyuk, "UV thermoresists: sub-100-nm imaging without proximity effects," Proc. SPIE 3676, 786-793 (1999).

12. G. H. Chapman, Y. Tu, and J. Peng, "Wavelength invariant Bi/In thermal resist as a Si anisotropic etch masking layer and direct-write photomask material," Proc. SPIE 5039, 472-483 (2003)

13. S. C. Chapra and R. P. Canale, Numerical Methods For Engineers: With Software and Programming, 4th ed., pp. 707-709, McGrawHill, New York (2002).

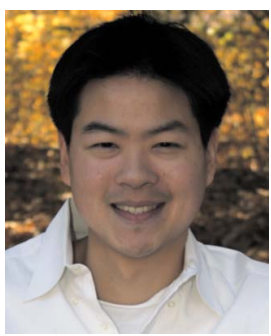

Saul Lee is a chemical engineering doctoral student at the University of Texas at Austin. $\mathrm{He}$ received his $\mathrm{BS}$ in chemical engineering from Georgia Institute of Technology in 2004. His primary research interests include DEL materials modeling and development, and modeling of self-assembly dynamics of photolithographically patterned biosensor arrays.

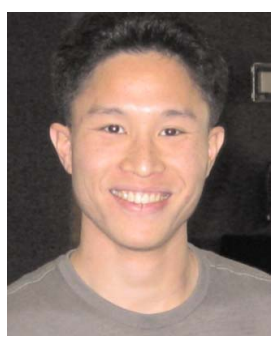

Kane Jen is a graduate student in the Department of Chemical Engineering at the University of Texas at Austin. He received his BS in chemical engineering from the University of Texas at Austin in 2000. From 2000 to 2004, Jen was employed by Intel Corporation as lithography process engineer in Portland, Oregon. He enrolled in the $\mathrm{PhD}$ program in chemical engineering at the University of Texas at Austin in August 2004.

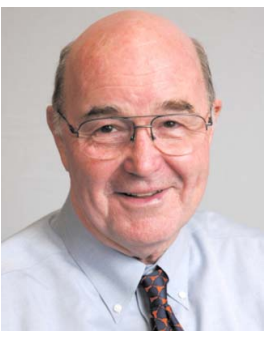

C. Grant Willson received his $\mathrm{BS}$ and $\mathrm{PhD}$ in organic chemistry from the University of California at Berkeley, and his MS in organic chemistry from San Diego State University. He came to the University of Texas from his position as an IBM Fellow and manager of the Polymer Science and Technology area at the IBM Almaden Research Center in San Jose, California. He has a joint appointment in the Departments of Chemistry and Chemical Engineering and holds the Rashid Engineering Regents Chair. He is the coauthor of more than 300 journal papers, is the editor and author of several books, and is coinventor on more than 25 issued patents. His research can be characterized as the design and synthesis of functional organic materials with emphasis on materials for microelectronics. He has received numerous awards for his work and is a member of the National Academy of Engineering.

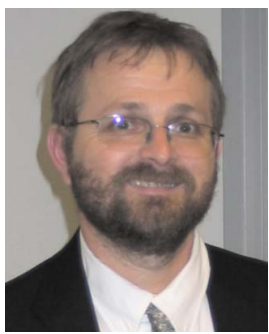

Jeffrey Byers received his BA in chemistry from the University of Arizona and $\mathrm{PhD}$ in physical chemistry from the University of Texas at Austin. He was a research scientist at KLA-Tencor before joining SEMATECH in 2006. His research interests include doubleexposure material development for sub$0.25 k_{1}$ imaging and simulation of feasibility for new material designs.

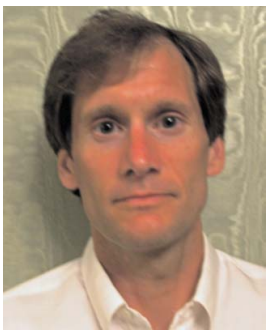

Paul Zimmerman received his BS, MS, and $\mathrm{PhD}$ in chemistry from the University of Pittsburgh, and also completed an MBA in 2000 at Arizona State University. He has worked for Intel Corporation since 1994. From 2001 to 2004, he spent three years on assignment at International SEMATECH developing materials for 157 and $193 \mathrm{~nm}$ immersion and EUV lithography. While on assignment at International SEMATECH, he authored over 30 published works and received three patents. Zimmerman recently finished an assignment at IMEC to aid in the development of high $\mathrm{k}$ dielectrics for the 22-nm node and beyond. He is also focused on development of non-Si nanotechnologies for low-power, high-performance logic applications. These include Ge for pMOS and ?III/V materials for nMOS. While at IMEC, Zimmerman and the Ge program team developed the highest performance Ge devices currently known. Most recently, he was assigned to the project manager's position at SEMATECH to develop high-index, double-exposure and nonchemically amplified resist materials for 193-nm immersion lithography.

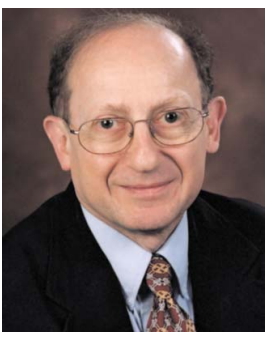

Nicholas J. Turro is the William P. Schweitzer Professor of Chemistry at Columbia University, where he has been since 1964 $\mathrm{He}$ received a BA in chemistry from Wesleyan University before going to Caltech for his $\mathrm{PhD}$ in chemistry in 1960 . He is the author of Modern Molecular Photochemistry, a standard text in the field, and has published over 800 research papers in established scientific journals. He pioneered the use of information technologies and computers for the enhancement and enrichment of undergraduate education in chemistry. His educational ideas and products are employed at universities and colleges across the nation. These accomplishments have been recognized by his selection as a Distinguished Teacher Scholar by the National Science Foundation in 2002, by Columbia University with a Presidential Teaching Award, and by the American Chemical Society with the Pimentel Award in Chemical Education. $\mathrm{He}$ is a member of the National Academy of Sciences and the American Academy of Arts and Sciences. 